\title{
The Maximum Spectral Radius of Graphs Without Friendship Subgraphs
}

\author{
Sebastian Cioabă ${ }^{*}$ \\ Department of Mathematical Sciences \\ University of Delaware \\ Newark, DE, U.S.A. \\ cioaba@udel.edu
}

\author{
Lihua Feng $^{\dagger}$ \\ School of Mathematics and Statistics \\ Central South University \\ New Campus, Changsha, Hunan, P.R. China \\ fenglh@163.com
}

\author{
Michael Tait ${ }^{\ddagger}$ \\ Department of Mathematics and Statistics \\ Villanova University \\ Villanova, PA, U.S.A. \\ michael.tait@villanova.edu \\ Xiao-Dong Zhang ${ }^{\S}$
}

School of Mathematical Sciences, MOE-LSC, SHL-MAC

Shanghai Jiao Tong University

Shanghai, P. R. China

xiaodong@sjtu.edu.cn

Submitted: Nov 29, 2019; Accepted: Oct 16, 2020; Published: Oct 30, 2020

(C) The authors. Released under the CC BY-ND license (International 4.0).

\begin{abstract}
A graph on $2 k+1$ vertices consisting of $k$ triangles which intersect in exactly one common vertex is called a $k$-friendship graph and denoted by $F_{k}$. This paper determines the graphs of order $n$ that have the maximum (adjacency) spectral radius among all graphs containing no $F_{k}$, for $n$ sufficiently large.
\end{abstract}

Mathematics Subject Classifications: 05C50; 05C38

${ }^{*}$ S. M. Cioabă is partially supported by NSF grants DMS-1600768 and CIF-1815922 and a JSPS Fellowship.

${ }^{\dagger}$ L. Feng is partially supported by NSFC (Nos. 11871479, 11671402), Hunan Provincial Natural Science Foundation (2016JJ2138, 2018JJ2479) and Mathematics and Interdisciplinary Sciences Project of CSU.

${ }^{\ddagger}$ M. Tait is partially supported by NSF grant DMS-2011553.

${ }^{\S}$ X.-D. Zhang is partially supported by NSFC (Nos. 11971311, 11531001); the Montenegrin-Chinese Science and Technology Cooperation Project (No.3-12) (Corresponding author). 


\section{Introduction}

In this paper, we consider only simple and undirected graphs. Let $G$ be a simple connected graph with vertex set $V(G)=\left\{v_{1}, \ldots, v_{n}\right\}$ and edge set $E(G)=\left\{e_{1}, \ldots, e_{m}\right\}$. Let $d\left(v_{i}\right)$ ( or $d_{G}\left(v_{i}\right)$ ) be the degree of a vertex $v$ in $G$. The adjacency matrix of $G$ is $A(G)=\left(a_{i j}\right)_{n \times n}$ with $a_{i j}=1$ if two vertices $v_{i}$ and $v_{j}$ are adjacent in $G$, and $a_{i j}=0$ otherwise. The largest eigenvalue of $A(G)$, denoted by $\lambda(G)$ or $\lambda_{1}(G)$, is called the spectral radius of $G$. The spectral radius of a graph gives some information about how dense the graph is. For example, it is well-known that the average degree of $G$ is at most $\lambda_{1}(G)$ which is at most the maximum degree of $G$.

In spectral graph theory, Brualdi and Solheid [5] proposed the following problem: Given a set of graphs, try to find a tight upper bound for the spectral radius in this set and characterize all extremal graphs. This problem is widely studied in the literature for many classes of graphs, such as graphs with a given number of cut vertices [3], diameter [8, 18], radius [18], domination number [29], size [28], Euler genus [9], and clique or independence number [30], and additionally for subgraphs of the hypercube [4].

The following problem regarding the adjacency spectral radius was proposed in [25]: What is the maximum spectral radius of a graph $G$ on $n$ vertices without a subgraph isomorphic to a given graph $F$ ? Fiedler and Nikiforov [13] obtained tight sufficient conditions for graphs to be Hamiltonian or traceable. Additionally, Nikiforov obtained spectral strengthenings of Turán's theorem [24] and the Kővari-Sós-Turán theorem [22] when the forbidden graphs are complete or complete bipartite respectively. This motivates further study for such question, see [12, 13, 20, 23, 25].

The Turán number of a graph $F$ is the maximum number of edges that may be in an $n$-vertex graph without a subgraph isomorphic to $F$, and is denoted by $\operatorname{ex}(n, F)$. A graph on $n$ vertices with no subgraph $F$ and with $\operatorname{ex}(n, F)$ edges is called an extremal graph for $F$ and we denote by $\operatorname{Ex}(n, F)$ the set of all extremal graphs on $n$ vertices for $F$. Understanding $\operatorname{ex}(n, F)$ and $\operatorname{Ex}(n, F)$ for various graphs $F$ is a cornerstone of extremal graph theory (see $[2,7,11,14,15,17,27]$ for surveys).

A graph on $2 k+1$ vertices consisting of $k$ triangles which intersect in exactly one common vertex is called a $k$-friendship graph (also known as a $k$-fan) and denoted by $F_{k}$.

In [10], the following result is proved.

Theorem 1. [10] For every $k \geqslant 1$, and for every $n \geqslant 50 k^{2}$, if a graph $G$ of order $n$ satisfies $e(G)>\operatorname{ex}\left(n, F_{k}\right)$, then $G$ contains a copy of a $k$-friendship graph, where

$$
\operatorname{ex}\left(n, F_{k}\right)=\left\lfloor\frac{n^{2}}{4}\right\rfloor+ \begin{cases}k^{2}-k & \text { if } k \text { is odd }, \\ k^{2}-\frac{3}{2} k & \text { if } k \text { is even. }\end{cases}
$$

The extremal graphs $G_{n, k}^{i}(i=1,2)$ of Theorem 1 are as follows. For odd $k$ (where $n \geqslant 4 k-1) G_{n, k}^{1}$ is constructed by taking a complete bipartite graph with color classes of size $\left\lceil\frac{n}{2}\right\rceil$ and $\left\lfloor\frac{n}{2}\right\rfloor$ and embedding two vertex disjoint copies of $K_{k}$ in one side. For even $k$ (where now $n \geqslant 4 k-3) G_{n, k}^{2}$ is constructed by taking a complete equi-bipartite graph and embedding a graph with $2 k-1$ vertices, $k^{2}-\frac{3}{2} k$ edges with maximum degree $k-1$ in one side. The graphs $G_{n, k}^{1}$ is unique up to isomorphism, but $G_{n, k}^{2}$ is not. 
Our goal is to give the spectral counterpart of Theorem 1 . As the case $k=1$ is just Mantel's theorem, whose spectral version is also known (see [21]) so we consider $k \geqslant 2$. The main result of this paper is the following.

Theorem 2. Let $G$ be a graph of order $n$ that does not contain a copy of a $k$-friendship graph, $k \geqslant 2$. For sufficiently large $n$, if $G$ has the maximal spectral radius, then

$$
G \in \operatorname{Ex}\left(n, F_{k}\right)
$$

We note that one may form an equitable partition of a graph in $\operatorname{Ex}\left(n, F_{k}\right)$ and determine its spectral radius as the root of a degree 3 (if $k$ is odd) or degree 4 (if $k$ is even) polynomial. We at last point out that, during our proof, we use the triangle removal lemma, so it is difficult to present exactly how large we need our $n$ to be.

\section{Some Lemmas}

Let $G$ be a simple graph with matching number $\beta(G)$ and maximum degree $\Delta(G)$. For given two integers $\beta$ and $\Delta$, define $f(\beta, \Delta)=\max \{|E(G)|: \beta(G) \leqslant \beta, \Delta(G) \leqslant \Delta\}$. Chvátal and Hanson [6] obtained the following result.

Theorem 3 (Chvátal and Hanson [6]). For every two positive integers $\beta \geqslant 1$ and $\Delta \geqslant 1$, we have

$$
f(\beta, \Delta)=\Delta \beta+\left\lfloor\frac{\Delta}{2}\right\rfloor\left\lfloor\frac{\beta}{\lceil\Delta / 2\rceil}\right\rfloor \leqslant \Delta+\beta .
$$

We will frequently use the following special case proved by Abbott, Hanson and Sauer [1]:

$$
f(k-1, k-1)= \begin{cases}k^{2}-k & \text { if } k \text { is odd } \\ k^{2}-\frac{3}{2} k & \text { if } k \text { is even. }\end{cases}
$$

The extremal graphs are exactly those we embedded into the Turán graph $T_{n, 2}$ to obtain the extremal $F_{k}$-free graph $G_{n, k}^{i}(i=1,2)$.

Essential to our proof are the following two lemmas: the triangle removal lemma and a stability result of Füredi.

Lemma 4 (Triangle Removal Lemma $[10,14,26]$ ). For each $\varepsilon>0$, there exists an $N=N(\varepsilon)$ and $\delta>0$ such that every graph $G$ on $n$ vertices with $n \geqslant N$ with at most $\delta n^{3}$ triangles can be made triangle-free by removing at most $\varepsilon n^{2}$ edges.

Lemma 5 (Füredi [16]). Suppose that $K_{3} \nsubseteq G,|V(G)|=n, s>0$ and $e(G)=e\left(T_{n, 2}\right)-s$. Then there exists a bipartite subgraph $H, E(H) \subseteq E(G)$ such that $e(H) \geqslant e(G)-s$.

The following lemma is needed in the sequel.

Lemma 6. For any positive integer n, we have

$$
\frac{n}{2}-\sqrt{\left\lceil\frac{n}{2}\right\rceil\left\lfloor\frac{n}{2}\right\rfloor}<\frac{1}{n} .
$$




\section{The Proof of Theorem 2}

Let $\mathcal{G}_{n, k}$ be the set of all $F_{k}$-free graphs of order $n$. Let $G \in \mathcal{G}_{n, k}$ be a graph on $n$ vertices with maximum spectral radius. The aim of this section is to prove that $e(G)=\operatorname{ex}\left(n, F_{k}\right)$ for $n$ large enough.

First note that $G$ must be connected. Let $\lambda_{1}$ be the spectral radius of $G$ and let $\mathbf{x}$ be a positive eigenvector for it. We may normalize $\mathbf{x}$ so that it has maximum entry equal to 1 , and let $z$ be a vertex such that $\mathbf{x}_{z}=1$. We prove the theorem iteratively, giving successively better lower bounds on both $e(G)$ and the eigenvector entries of all of the other vertices, until finally we can show that $e(G)=\operatorname{ex}\left(n, F_{k}\right)$.

Let $H \in \operatorname{Ex}\left(n, F_{k}\right)$. Then since $G$ is the graph maximizing the spectral radius over all $F_{k}$-free graphs, in view of Theorem 1 , we must have

$$
\lambda_{1}(G) \geqslant \lambda_{1}(H) \geqslant \frac{\mathbf{1}^{T} A(H) \mathbf{1}}{\mathbf{1}^{T} \mathbf{1}}=2 \frac{\left\lceil\frac{n}{2}\right\rceil\left\lfloor\frac{n}{2}\right\rfloor+f(k-1, k-1)}{n}>\frac{n}{2} .
$$

The proof of Theorem 2 is outlined as follows.

- We give a lower bound on $e(G)$ as a function of $\lambda_{1}$ and the number $t$ of triangles in $G$, which on first approximation gives a bound of roughly $\frac{n^{2}}{4}-O(k n)$.

- Using the triangle removal lemma and Füredi's stability result, we show that $G$ has a very large maximum cut.

- We show that no vertex has many neighbors on its side of the partition, and then we refine this by considering eigenvector entries to show that in fact no vertex has more than a constant number of neighbors on its side of the partition.

- We show that no vertices have degree much smaller than $\frac{n}{2}$, and this allows us to refine our lower bound on both $e(G)$ and on the eigenvector entry of each vertex.

- Once we know that all vertices have eigenvector entry very close to 1 , we may show that the partition is balanced. This shows that $G$ can be converted to a graph in $\operatorname{Ex}\left(n, F_{k}\right)$ by adding or removing a constant number of edges, and this allows us to show that $e(G)=\operatorname{ex}\left(n, F_{k}\right)$.

We now proceed with the details. First we prove a lemma which gives a lower bound on $e(G)$ in terms of $\lambda_{1}$ and the number of triangles in $G$.

Lemma 7. If $G$ has $t$ triangles, then

$$
e(G) \geqslant \lambda_{1}^{2}-\frac{3 t}{\lambda_{1}}
$$

Proof. Let $\lambda_{1}$ be the spectral radius of $G$ and let $\mathbf{x}$ be a positive eigenvector scaled such that it has maximum entry equal to 1 , and let $z$ be a vertex with maximum eigenvector 
entry i.e., $\mathbf{x}_{z}=1$. Then $\lambda_{1} \mathbf{x}_{u}=\sum_{v \sim u} \mathbf{x}_{v}$ and $\lambda_{1}^{2} \mathbf{x}_{u}=\sum_{v \sim u} \lambda_{1} \mathbf{x}_{v}=\sum_{v \sim u} \sum_{w \sim v} \mathbf{x}_{w}$. We consider the following triple sum:

$$
\sum_{u \in V} \lambda_{1}^{2} \mathbf{x}_{u}=\sum_{u \in V} \sum_{v \sim u} \sum_{w \sim v} \mathbf{x}_{w}
$$

The sum counts over all ordered walks on three vertices (with possible repetition), and is weighted by the eigenvector entry of the last vertex. Instead of summing over ordered triples of vertices, we count by considering the first edge in the walk. If a given walk has first edge $u v$, then $\mathbf{x}_{w}$ will be counted by this edge exactly once if $w$ is adjacent to exactly one of $u$ or $v$ and exactly twice if $\{u, v, w\}$ forms a triangle. Therefore, the sum is equal to

$$
\begin{aligned}
\sum_{u v \in E}\left(2 \sum_{\substack{w u \in E \\
w v \in E}} \mathbf{x}_{w}+\sum_{\substack{w u \in E \\
w v \notin E}} \mathbf{x}_{w}+\sum_{\substack{w v \in E \\
w u \notin E}} \mathbf{x}_{w}\right) & =\sum_{u v \in E}\left(\sum_{\substack{w u \in E \\
w v \in E}} \mathbf{x}_{w}+\sum_{\substack{w u \in E \\
w v \notin E}} \mathbf{x}_{w}+\sum_{w v \in E} \mathbf{x}_{w}\right) \\
& \leqslant \sum_{w v \in E}\left(\sum_{\substack{w u \in E \\
w v \in E}} \mathbf{x}_{w}+\sum_{w v \notin E} \mathbf{x}_{w}+\sum_{w v \in E} \mathbf{x}_{w}\right) \\
& \leqslant \sum_{u v \in E}\left(\sum_{\substack{w u \in E \\
w v \in E}} \mathbf{x}_{w}+\sum_{w \in V} \mathbf{x}_{w}\right) \\
& \leqslant \sum_{u v \in E}\left(\sum_{\substack{w u \in E \\
w v \in E}} 1+\sum_{w \in V} \mathbf{x}_{w}\right) \\
& =3 t+e(G) \sum_{w \in V} \mathbf{x}_{w} .
\end{aligned}
$$

Hence

On the other hand,

$$
e(G) \geqslant \lambda_{1}^{2}-\frac{3 t}{\sum_{w \in V} \mathbf{x}_{w}}
$$

$$
\lambda_{1}=\lambda_{1} \mathbf{x}_{z}=\sum_{w \sim z} \mathbf{x}_{w} \leqslant \sum_{w \in V} \mathbf{x}_{w}
$$

Therefore

$$
e(G) \geqslant \lambda_{1}^{2}-\frac{3 t}{\sum_{w \in V} \mathbf{x}_{w}} \geqslant \lambda_{1}^{2}-\frac{3 t}{\lambda_{1}}
$$

So the assertion holds.

Corollary 8. If the number of triangles of $G$ is $t$, then

$$
e(G) \geqslant \lambda_{1}^{2}-\frac{6 t}{n}
$$


Proof. In view of inequality (1), and the function $f(x)=\lambda_{1}^{2}-\frac{3 t}{x}$ is strictly increasing with respect to $x$, the assertion follows.

Lemma 9. Suppose the matching number of a graph $H$ of order $n$ is at most $k-1$. Then $e(H) \leqslant k n$, i.e., $\operatorname{ex}\left(n, M_{k}\right) \leqslant k n$, where $M_{k}$ is a matching of size $k$.

Proof. By Theorem 3, $e(H) \leqslant f(k-1, n-1) \leqslant(k-1)(n-1+1)<k n$.

Lemma 10. Let $\varepsilon$ and $\delta$ be fixed positive constants with $\delta<\frac{1}{10(k+1)^{2}}, \varepsilon<\frac{\delta^{2}}{16}$. There exists an $N(\varepsilon, \delta, k)$ such that $G$ has a partition $V=S \cup T$ which gives a maximum cut, and

$$
e(S, T) \geqslant\left(\frac{1}{4}-\varepsilon\right) n^{2}
$$

for $n \geqslant N(\varepsilon, \delta, k)$. Furthermore

$$
\left(\frac{1}{2}-\sqrt{\varepsilon}\right) n \leqslant|S|,|T| \leqslant\left(\frac{1}{2}+\sqrt{\varepsilon}\right) n
$$

Proof. Since $G$ is $F_{k}$-free, the neighborhood of any vertex does not have $M_{k}$ (a matching of size $k$ ) as a subgraph. Thus by Lemma 9, we can obtain the following upper bound for the number of triangles,

$$
3 t=\sum_{v \in V(G)} e(G[N(v)]) \leqslant \sum_{v \in V(G)} \operatorname{ex}\left(d(v)+1, M_{k}\right) \leqslant \sum_{v \in V(G)} \operatorname{ex}\left(n, M_{k}\right) \leqslant \sum_{v \in V(G)} k n=k n^{2} .
$$

This gives $t \leqslant \frac{k n^{2}}{3}$. So $t \leqslant \frac{k}{3 n} n^{3} \leqslant \delta n^{3}$ for $n \geqslant N_{2} \geqslant \frac{k}{3 \delta}$. From Corollary 8 , we obtain

$$
e(G) \geqslant \frac{n^{2}}{4}-2 k n
$$

By Lemma 4, there exists an $N_{1}(\varepsilon, k)$ such that the graph $G_{1}$ obtained from $G$ by deleting at most $\frac{1}{10} \varepsilon n^{2}$ edges is $K_{3}$-free. For $N=\max \left\{N_{1}, N_{2}\right\}$, the size of the graph $G_{1}$ of order $n \geqslant N$ satisfies $e\left(G_{1}\right) \geqslant e(G)-\frac{1}{10} \varepsilon n^{2}$. Note that $e\left(G_{1}\right) \leqslant e\left(T_{n, 2}\right)$ by Turán's Theorem. Define

$$
s \triangleq e\left(T_{n, 2}\right)-e\left(G_{1}\right) \geqslant 0 .
$$

By Lemma $5, G_{1}$ contains a bipartite subgraph $G_{2}$ such that $e\left(G_{2}\right) \geqslant e\left(G_{1}\right)-s$. Hence, for $n$ sufficiently large, we have

$$
\begin{aligned}
e\left(G_{2}\right) & \geqslant e\left(G_{1}\right)-s \\
& =2 e\left(G_{1}\right)-e\left(T_{n, 2}\right) \\
& \geqslant 2 e(G)-e\left(T_{n, 2}\right)-\frac{1}{5} \varepsilon n^{2} \\
& \geqslant 2\left(\frac{n^{2}}{4}-2 k n\right)-\frac{n^{2}}{4}-\frac{1}{5} \varepsilon n^{2}
\end{aligned}
$$




$$
\geqslant\left(\frac{1}{4}-\varepsilon\right) n^{2}
$$

Therefore, $G$ has a partition $V=S \cup T$ which gives a maximum cut such that

$$
e(S, T) \geqslant e\left(G_{2}\right) \geqslant\left(\frac{1}{4}-\varepsilon\right) n^{2} .
$$

Furthermore, without loss of generality, we may assume that $|S| \leqslant|T|$. If $|S|<\left(\frac{1}{2}-\sqrt{\varepsilon}\right) n$, then $|T|=n-|S|>\left(\frac{1}{2}+\sqrt{\varepsilon}\right) n$. So

$$
e(S, T) \leqslant|S||T|<\left(\frac{1}{2}-\sqrt{\varepsilon}\right) n\left(\frac{1}{2}+\sqrt{\varepsilon}\right) n=\left(\frac{1}{4}-\varepsilon\right) n^{2},
$$

which contradicts to Eq. (3). Therefore it follows that

$$
\left(\frac{1}{2}-\sqrt{\varepsilon}\right) n \leqslant|S|,|T| \leqslant\left(\frac{1}{2}+\sqrt{\varepsilon}\right) n
$$

Hence the assertion holds.

Lemma 11. Let $k \geqslant 2$. Denote by

$$
L:=\left\{v: d(v) \leqslant\left(\frac{1}{2}-\frac{1}{4(k+1)}\right) n\right\} .
$$

Then

$$
|L| \leqslant 16 k^{2} .
$$

Proof. Suppose that $|L|>16 k^{2}$. Then let $L^{\prime} \subseteq L$ with $\left|L^{\prime}\right|=16 k^{2}$. Then it follows that

$$
\begin{aligned}
e\left(G-L^{\prime}\right) & \geqslant e(G)-\sum_{v \in L^{\prime}} d(v) \\
& \geqslant \frac{n^{2}}{4}-2 k n-16 k^{2}\left(\frac{1}{2}-\frac{1}{4(k+1)}\right) n \\
& >\frac{\left(n-16 k^{2}\right)^{2}}{4}+k^{2} .
\end{aligned}
$$

for $n$ a sufficiently large constant depending only on $k$, where the second inequality is by (2). Hence by Theorem $1, G-L^{\prime}$ contains $F_{k}$, which implies that $G$ contains $F_{k}$. So the assertion holds.

We will also need the following lemma which can be proved by induction or double counting.

Lemma 12. Let $A_{1}, \cdots, A_{p}$ be $p$ finite sets. Then

$$
\left|A_{1} \cap A_{2} \cap \cdots \cap A_{p}\right| \geqslant \sum_{i=1}^{p}\left|A_{i}\right|-(p-1)\left|\bigcup_{i=1}^{p} A_{i}\right| .
$$


For a vertex $v$, let $d_{S}(v)=|N(v) \cap S|$ and $d_{T}(v)=|N(v) \cap T|$, and let

$$
W:=\left\{v \in S: d_{S}(v) \geqslant \delta n\right\} \cup\left\{v \in T: d_{T}(v) \geqslant \delta n\right\}
$$

be the set of vertices that have many neighbors which are not in the cut. Let $L$ be as in Lemma 11, that is

$$
L=\left\{v: d(v) \leqslant\left(\frac{1}{2}-\frac{1}{4(k+1)}\right) n\right\}
$$

Next we show that actually $W$ and $L$ are empty.

Lemma 13. For the above $W$, we have

$$
|W|<\frac{2 \varepsilon}{\delta} n+\frac{2 k^{2}}{\delta n}
$$

and $W \backslash L$ is empty.

Proof. Note that $e(S, T) \geqslant\left(\frac{1}{4}-\varepsilon\right) n^{2}$ by Lemma 10 , and $e(G) \leqslant \operatorname{ex}\left(n, F_{k}\right) \leqslant \frac{n^{2}}{4}+k^{2}$ by Theorem 1. Hence

$$
e(S)+e(T)=e(G)-e(S, T) \leqslant \frac{n^{2}}{4}+k^{2}-\left(\frac{1}{4}-\varepsilon\right) n^{2}=\varepsilon n^{2}+k^{2} .
$$

On the other hand, if we let $W_{1}=W \cap S$ and $W_{2}=W \cap T$, then we deduce

$$
2 e(S)=\sum_{u \in S} d_{S}(u) \geqslant \sum_{u \in W_{1}} d_{S}(u) \geqslant\left|W_{1}\right| \delta n, \quad 2 e(T)=\sum_{u \in T} d_{T}(u) \geqslant \sum_{u \in W_{2}} d_{T}(u) \geqslant\left|W_{2}\right| \delta n .
$$

So

$$
e(S)+e(T) \geqslant\left(\left|W_{1}\right|+\left|W_{2}\right|\right) \frac{\delta n}{2}=\frac{|W| \delta n}{2} .
$$

By (5) and (6), we get

$$
\frac{|W| \delta n}{2} \leqslant \varepsilon n^{2}+k^{2}
$$

i.e.,

$$
|W| \leqslant \frac{2\left(\varepsilon n^{2}+k^{2}\right)}{\delta n} .
$$

Suppose that $W \backslash L \neq \emptyset$. We now prove that this is impossible.

Let $L_{1}=L \cap S$ and $L_{2}=L \cap T$. Without loss of generality, there exists a vertex $u \in W_{1} \backslash L_{1}$. Since $S$ and $T$ form a maximum cut, $d_{T}(u) \geqslant \frac{1}{2} d(u)$. On the other hand, $u \notin L$ because $u \in W_{1} \backslash L_{1}$. Therefore $d(u) \geqslant\left(\frac{1}{2}-\frac{1}{4(k+1)}\right) n$. So

$$
d_{T}(u) \geqslant \frac{1}{2} d(u) \geqslant\left(\frac{1}{4}-\frac{1}{8(k+1)}\right) n
$$


On the other hand, $|L| \leqslant 16 k^{2}$. Hence, for fixed $\delta<\frac{1}{10(k+1)^{2}}, \varepsilon<\frac{\delta^{2}}{16}$ and sufficiently large $n$, we have

$$
|S \backslash(W \cup L)| \geqslant\left(\frac{1}{2}-\sqrt{\varepsilon}\right) n-\delta n-\frac{2 k^{2}}{\delta n}-16 k^{2} \geqslant\left(\frac{1}{2}-\sqrt{\varepsilon}-\delta\right) n-18 k^{2} \geqslant k .
$$

Suppose that $u$ is adjacent to $k$ vertices $u_{1}, \ldots, u_{k}$ in $S \backslash(W \cup L)$. Since $u_{i} \notin L$, we have $d\left(u_{i}\right) \geqslant\left(\frac{1}{2}-\frac{1}{4(k+1)}\right) n$. On the other hand, $d_{S}\left(u_{i}\right) \leqslant \delta n$ by $u_{i} \notin W$. So $d_{T}\left(u_{i}\right)=d\left(u_{i}\right)-d_{S}\left(u_{i}\right) \geqslant\left(\frac{1}{2}-\frac{1}{4(k+1)}-\delta\right) n$. By Lemma 12, we have

$$
\begin{aligned}
& \left|N_{T}(u) \cap N_{T}\left(u_{1}\right) \cap \cdots \cap N_{T}\left(u_{k}\right)\right| \\
\geqslant & \left|N_{T}(u)\right|+\left|N_{T}\left(u_{1}\right)\right|+\ldots+\left|N_{T}\left(u_{k}\right)\right|-k\left|N_{T}(u) \cup N_{T}\left(u_{1}\right) \cup \cdots \cup N_{T}\left(u_{k}\right)\right| \\
\geqslant & d_{T}(u)+d_{T}\left(u_{1}\right)+\cdots+d_{T}\left(u_{k}\right)-k|T| \\
\geqslant & \left(\frac{1}{4}-\frac{1}{8(k+1)}\right) n+\left(\frac{1}{2}-\frac{1}{4(k+1)}-\delta\right) n \cdot k-k\left(\frac{1}{2}+\sqrt{\varepsilon}\right) n \\
= & \left(\frac{1}{8(k+1)}-k \delta-k \sqrt{\varepsilon}\right) n>k
\end{aligned}
$$

for sufficiently large $n$, where the last inequality is from $\delta<\frac{1}{10(k+1)^{2}}$ and $\varepsilon<\frac{\delta^{2}}{16}$. So there exist $k$ vertices $v_{1}, \ldots v_{k}$ in $T$ such that the induced subgraph by two partitions $\left\{u_{1}, \ldots, u_{k}\right\}$ and $\left\{v_{1}, \ldots, v_{k}\right\}$ is complete bipartite. It follows that $G$ contains $F_{k}$, this is a contradiction. Therefore $u$ is adjacent to at most $k-1$ vertices in $S \backslash(W \cup L)$. Hence, in view of $\varepsilon<\frac{\delta^{2}}{16}$, we have

$$
\begin{aligned}
d_{S}(u) & \leqslant|W|+|L|+k-1 \\
& <\frac{2 \varepsilon}{\delta} n+\frac{2 k^{2}}{\delta n}+16 k^{2}+k-1 \\
& <\frac{2 \delta}{3} n+\frac{2 k^{2}}{\delta n}+17 k^{2} \\
& <\delta n
\end{aligned}
$$

for sufficiently large $n$. This is a contradiction to the fact that $u \in W$. Similarly, there is no vertex $u \in W_{2} \backslash L_{2}$, Hence $W \backslash L=\emptyset$.

Lemma 14. $L$ is empty, and both $G[S]$ and $G[T]$ are $K_{1, k}$ and $M_{k}$-free.

Proof. We will prove the result from the following two claims.

Claim 1: There exist independent sets $I_{S} \subseteq S$ and $I_{T} \subseteq T$ such that

$$
\left|I_{S}\right| \geqslant|S|-18 k^{2}, \quad \text { and }\left|I_{T}\right| \geqslant|T|-18 k^{2} .
$$

Indeed, let $u_{1}, \ldots, u_{2 k} \in S \backslash L$. Then $u_{i} \notin L$ which implies

$$
d\left(u_{i}\right) \geqslant\left(\frac{1}{2}-\frac{1}{4(k+1)}\right) n \text {. }
$$


By Lemma $13, d_{S}\left(u_{i}\right) \leqslant \delta n$. Hence

$$
d_{T}\left(u_{i}\right)=d\left(u_{i}\right)-d_{S}\left(u_{i}\right) \geqslant\left(\frac{1}{2}-\frac{1}{4(k+1)}-\delta\right) n .
$$

Furthermore, by Lemma 12, we have

$$
\begin{aligned}
\left|\bigcap_{i=1}^{2 k} N_{T}\left(u_{i}\right)\right| & \geqslant \sum_{i=1}^{2 k}\left|N_{T}\left(u_{i}\right)\right|-(2 k-1)\left|\bigcup_{i=1}^{2 k} N_{T}\left(u_{i}\right)\right| \\
& \geqslant\left(\frac{1}{2}-\frac{1}{4(k+1)}-\delta\right) n \cdot 2 k-(2 k-1)\left(\frac{1}{2}+\sqrt{\varepsilon}\right) n \\
& =\left(\frac{1}{2(k+1)}-2 k \delta-(2 k-1) \sqrt{\varepsilon}\right) n \\
& >k
\end{aligned}
$$

for sufficiently large $n$. Hence there exist $k$ vertices $v_{1}, \ldots, v_{k}$ such that the induced subgraph by two partitions $\left\{u_{1}, \ldots, u_{2 k}\right\}$ and $\left\{v_{1}, \ldots, v_{k}\right\}$ is a complete bipartite graph. So $G[S \backslash L]$ is both $K_{1, k}$ and $M_{k}$-free, otherwise $G$ contains $F_{k}$, i.e., $u u_{1} v_{1}, \ldots u u_{k} v_{k}$ for $d(u) \geqslant k$, or $v_{1} u_{1} u_{2}, \ldots, v_{1} u_{2 k-1} u_{2 k}$ for $\left\{u_{1} u_{2}, \ldots u_{2 k-1} u_{2 k}\right\}$ being a matching of size $k$. Hence the maximum degree and the maximum matching number of $G[S \backslash L]$ are at most $k-1$, respectively. By Theorem 3,

$$
e(G[S \backslash L]) \leqslant f(k-1, k-1) .
$$

The same argument gives

$$
e(G[T \backslash L]) \leqslant f(k-1, k-1) .
$$

Since $G[S \backslash L]$ has at most $f(k-1, k-1)$ edges, then the subgraph obtained from $G[S \backslash L]$ by deleting one vertex of each edge in $G[S \backslash L]$ contains no edges, which is an independent set of $G[S \backslash L]$. So there exists an independent set $I_{S} \subseteq S$ such that

$$
\left|I_{S}\right| \geqslant|S \backslash L|-f(k-1, k-1) \geqslant|S|-k\left(k-\frac{3}{2}\right)-16 k^{2} \geqslant|S|-18 k^{2} .
$$

The same argument gives that there is an independent set $I_{T} \subseteq T$ with

$$
\left|I_{T}\right| \geqslant|T|-18 k^{2}
$$

So Claim 1 holds.

Recall that $z$ is a vertex with maximum eigenvector entry. Since $\mathbf{x}_{z}=1$, and

$$
d(z) \geqslant \sum_{w \sim z} \mathbf{x}_{w}=\lambda_{1} \mathbf{x}_{z}=\lambda_{1} \geqslant \frac{n}{2}
$$

Hence $z \notin L$. 
Without loss of generality, we may assume that $z \in S$. Since the maximum degree in the induced subgraph $G[S \backslash L]$ is at most $k-1$ (containing no $K_{1, k}$ ), from Lemma 11, we have $|L| \leqslant 16 k^{2}$ and

$$
d_{S}(z)=d_{S \cap L}(z)+d_{S \backslash L}(z) \leqslant k-1+16 k^{2} \leqslant 17 k^{2} .
$$

Therefore, by Claim 1, we have

$$
\begin{aligned}
\lambda_{1} & =\lambda_{1} \mathbf{x}_{z}=\sum_{v \sim z} \mathbf{x}_{v} \\
& =\sum_{v \sim z, v \in S} \mathbf{x}_{v}+\sum_{v \sim z, v \in T} \mathbf{x}_{v} \\
& =\sum_{v \sim z, v \in S} \mathbf{x}_{v}+\sum_{v \sim z, v \in I_{T}} \mathbf{x}_{v}+\sum_{v \sim z, v \in T \backslash I_{T}} \mathbf{x}_{v} \\
& \leqslant d_{S}(z)+\sum_{v \in I_{T}} \mathbf{x}_{v}+\sum_{v \in T \backslash I_{T}} 1 \\
& \leqslant 17 k^{2}+\sum_{v \in I_{T}} \mathbf{x}_{v}+|T|-\left|I_{T}\right| \\
& \leqslant \sum_{v \in I_{T}} \mathbf{x}_{v}+17 k^{2}+18 k^{2} \\
& \leqslant \sum_{v \in I_{T}} \mathbf{x}_{v}+35 k^{2} .
\end{aligned}
$$

So

$$
\sum_{v \in I_{T}} \mathbf{x}_{v} \geqslant \lambda_{1}-35 k^{2}
$$

Claim 2: $L=\emptyset$.

By way of contradiction, assume that there is a vertex $v \in L$, i.e., $d(v) \leqslant\left(\frac{1}{2}-\frac{1}{4(k+1)}\right) n$. Consider the graph $G^{+}$with vertex set $V(G)$ and edge set $E\left(G^{+}\right)=E(G \backslash\{v\}) \cup\{v w$ : $\left.w \in I_{T}\right\}$. Note that adding a vertex incident with vertices in $I_{T}$ does not create any triangles, and so $G^{+}$is $F_{k}$-free. By (8), we have that

$$
\begin{aligned}
\lambda_{1}\left(G^{+}\right)-\lambda_{1}(G) & \geqslant \frac{\mathbf{x}^{T}\left(A\left(G^{+}\right)-A(G)\right) \mathbf{x}}{\mathbf{x}^{\mathbf{T}} \mathbf{x}}=\frac{2 \mathbf{x}_{v}}{\mathbf{x}^{T} \mathbf{x}}\left(\sum_{w \in I_{T}} \mathbf{x}_{w}-\sum_{u v \in E(G)} \mathbf{x}_{u}\right) \\
& \geqslant \frac{2 \mathbf{x}_{v}}{\mathbf{x}^{T} \mathbf{x}}\left(\lambda_{1}-35 k^{2}-d_{G}(v)\right) \\
& \geqslant \frac{2 \mathbf{x}_{v}}{\mathbf{x}^{T} \mathbf{x}}\left(\lambda_{1}-35 k^{2}-\left(\frac{1}{2}-\frac{1}{4(k+1)}\right) n\right) \\
& \geqslant \frac{2 \mathbf{x}_{v}}{\mathbf{x}^{T} \mathbf{x}}\left(\frac{n}{2}-35 k^{2}-\left(\frac{1}{2}-\frac{1}{4(k+1)}\right) n\right) \\
& =\frac{2 \mathbf{x}_{v}}{\mathbf{x}^{T} \mathbf{x}}\left(\frac{n}{4(k+1)}-35 k^{2}\right)>0,
\end{aligned}
$$


where the last step uses $n$ large enough and that if $v \in L$, then $d_{G}(v) \leqslant\left(\frac{1}{2}-\frac{1}{4 k+4}\right) n$. This contradicts $G$ has the largest spectral radius over all $F_{k}$-free graphs and so $L$ must be empty.

Next we may refine the structure of $G$.

Lemma 15. For $n$ and $k$ as before, we have

$$
\begin{gathered}
\frac{n}{2}-4 k \leqslant|S|,|T| \leqslant \frac{n}{2}+4 k, \\
e(G) \geqslant \frac{n^{2}}{4}-12 k^{2},
\end{gathered}
$$

and

$$
\frac{n}{2}-14 k^{2} \leqslant \delta(G) \leqslant \lambda_{1} \leqslant \Delta(G) \leqslant \frac{n}{2}+5 k .
$$

Proof. From Lemma 14, both $G[S]$ and $G[T]$ are $K_{1, k}$ and $M_{k}$-free, so we have $e(S)+$ $e(T) \leqslant 2 f(k-1, k-1)<2 k^{2}$. This means that the number of triangles in $G$ is bounded above by $2 k^{2} n$ since any triangle contains an edge of $E(S) \cup E(T)$. By Corollary 8, we have

$$
e(G) \geqslant \lambda_{1}^{2}-\frac{6 t}{n} \geqslant \frac{n^{2}}{4}-\frac{12 k^{2} n}{n}=\frac{n^{2}}{4}-12 k^{2} .
$$

Suppose that $|S| \leqslant \frac{n}{2}-4 k$, then $|T|=n-|S| \geqslant \frac{n}{2}+4 k$. Hence

$$
e(G)=e(S)+e(T)+e(S, T) \leqslant 2 k^{2}+|S||T| \leqslant 2 k^{2}+\left(\frac{n}{2}-4 k\right)\left(\frac{n}{2}+4 k\right)=\frac{n^{2}}{4}-14 k^{2}
$$

which contradicts to $e(G) \geqslant \frac{n^{2}}{4}-12 k^{2}$.

So we have

$$
\frac{n}{2}-4 k \leqslant|S|,|T| \leqslant \frac{n}{2}+4 k .
$$

Moreover, by Lemma 14, the maximum degree of $G[S]$ is at most $k-1$. This implies that

$$
\Delta(G) \leqslant \frac{n}{2}+4 k+k-1 \leqslant \frac{n}{2}+5 k .
$$

So

$$
\lambda_{1} \leqslant \Delta(G) \leqslant \frac{n}{2}+5 k .
$$

Furthermore, we claim that the minimum degree of $G$ is at least $\frac{n}{2}-14 k^{2}$. Otherwise, removing a vertex $v$ of minimum degree $d(v)$, we have

$$
\begin{aligned}
e(G-v) & =e(G)-d(v) \\
& \geqslant \frac{n^{2}}{4}-12 k^{2}-\left(\frac{n}{2}-14 k^{2}\right) \\
& =\frac{n^{2}}{4}-\frac{n}{2}+2 k^{2}
\end{aligned}
$$




$$
\begin{aligned}
& =\frac{(n-1)^{2}}{4}+k^{2}+k^{2}-\frac{1}{4} \\
& >\frac{(n-1)^{2}}{4}+k^{2},
\end{aligned}
$$

which implies $G-v$ contains $F_{k}$ by Theorem 1 .

Lemma 16. For all $u \in V(G)$, we have that $\mathbf{x}_{u} \geqslant 1-\frac{120 k^{2}}{n}$.

Proof. Without loss of generality, we may assume that $z \in S$. We consider the following two cases.

Case 1: $u \in S$. Then $d_{S}(u) \leqslant k^{2}$ as $e(G[S]) \leqslant k^{2}$. Hence we obtain

$$
\begin{aligned}
\left|N_{T}(u)\right|= & d_{T}(u)=d(u)-d_{S}(u) \geqslant \delta(G)-d_{S}(u) \geqslant \frac{n}{2}-14 k^{2}-k^{2} \\
\geqslant & \frac{n}{2}-15 k^{2} . \\
\left|N_{T}(u) \cap N_{T}(z)\right|= & \left|N_{T}(u)\right|+\left|N_{T}(z)\right|-\left|N_{T}(u) \cup N_{T}(z)\right| \geqslant 2 \delta_{T}(G)-|T| \\
\geqslant & 2\left(\frac{n}{2}-15 k^{2}\right)-\left(\frac{n}{2}+4 k\right) \geqslant \frac{n}{2}-34 k^{2} . \\
\lambda_{1} \mathbf{x}_{u}-\lambda_{1} \mathbf{x}_{z}= & \sum_{v \sim u, v \in T, v \sim z} \mathbf{x}_{v}+\sum_{v \sim u, v \in T, v \nsim z} \mathbf{x}_{v}+\sum_{v \sim u, v \in S} \mathbf{x}_{v} \\
& -\sum_{v \sim z, v \in T, v \sim u} \mathbf{x}_{v}-\sum_{v \sim z, v \in T, v \ngtr u} \mathbf{x}_{v}-\sum_{v \sim z, v \in S} \mathbf{x}_{v} \\
\geqslant & -\sum_{v \sim z, v \in T, v \not u} \mathbf{x}_{v}-\sum_{v \sim z, v \in S} \mathbf{x}_{v} 1-\sum_{v \sim z, v \in S} 1 \\
\geqslant & -\left(\sum_{v \sim z, v \in T, v \ngtr u} 1(z)-\left|N_{T}(u) \cap N_{T}(z)\right|\right)-d_{S}(z) \\
\geqslant & -\left(\left(\frac{n}{2}+5 k\right)-\left(\frac{n}{2}-34 k^{2}\right)\right)-k^{2} \\
\geqslant & -40 k^{2} .
\end{aligned}
$$

Therefore, for any $u \in S$, we have

$$
\mathbf{x}_{u} \geqslant 1-\frac{40 k^{2}}{\lambda_{1}}>1-\frac{40 k^{2}}{\frac{n}{2}}=1-\frac{80 k^{2}}{n}
$$

Case 2: $u \in T$. By (12),

$$
\lambda_{1} \mathbf{x}_{u}=\sum_{v \sim u} \mathbf{x}_{v} \geqslant \sum_{v \sim u, v \in S} \mathbf{x}_{v} \geqslant\left(1-\frac{80 k^{2}}{n}\right) d_{S}(u) .
$$

Since

$$
\frac{n}{2}-14 k^{2} \leqslant \delta(G) \leqslant d(u)=d_{S}(u)+d_{T}(u)
$$


and $d_{T}(u) \leqslant k$ as the maximum degree in $G[T]$ is at most $k-1$, we have $d_{S}(u) \geqslant$ $\frac{n}{2}-14 k^{2}-k \geqslant \frac{n}{2}-15 k^{2}$. Hence

$$
\begin{aligned}
\mathbf{x}_{u} & \geqslant \frac{\left(1-\frac{80 k^{2}}{n}\right) d_{S}(u)}{\lambda_{1}} \geqslant \frac{\left(1-\frac{80 k^{2}}{n}\right)\left(\frac{n}{2}-15 k^{2}\right)}{\frac{n}{2}+5 k} \\
& =\frac{\frac{n}{2}-55 k^{2}+\frac{1200 k^{4}}{n}}{\frac{n}{2}+5 k} \\
& =1-\frac{55 k^{2}+5 k-\frac{1200 k^{4}}{n}}{\frac{n}{2}+5 k} \\
& >1-\frac{120 k^{2}}{n} .
\end{aligned}
$$

From the above two cases, the result follows.

Using this refined bound on the eigenvector entries, we may show that the partition $V=S \cup T$ is balanced.

Lemma 17. The sets $S$ and $T$ have sizes as close as possible. That is

$$
|| S|-| T|| \leqslant 1
$$

Proof. Without loss of generality, we may assume that $|T| \geqslant|S|$. Denote

$$
\begin{aligned}
& S^{\prime}:=\{v \in S: N(v) \subseteq T\}, \\
& T^{\prime}:=\{v \in T: N(v) \subseteq S\} .
\end{aligned}
$$

Since $e(G[S]) \leqslant k^{2}$, there exist at most $2 k^{2}$ vertices in $S$ having a neighbor in $S$. Hence

$$
\left|S^{\prime}\right| \geqslant|S|-2 k^{2} \text {. }
$$

Similarly,

$$
\left|T^{\prime}\right| \geqslant|T|-2 k^{2}
$$

Let $C \subseteq T^{\prime}$ be a set having $|T|-|S|$ vertices, which exists since, from (9), $|T|-|S| \leqslant 8 k$ and $\left|T^{\prime}\right| \geqslant|T|-2 k^{2} \geqslant \frac{n}{2}-4 k-2 k^{2}>8 k$. Then $G \backslash C$ is a graph on $2|S|$ vertices such that

$$
e(G)-e(C, S)=e(G \backslash C) \leqslant \operatorname{ex}\left(2|S|, F_{k}\right) \leqslant \frac{(2|S|)^{2}}{4}+f(k-1, k-1) .
$$

Hence

$$
e(G) \leqslant|S|^{2}+|C||S|+f(k-1, k-1)=|S||T|+f(k-1, k-1) .
$$

Let $B=K_{|S|,|T|}$ be the complete bipartite graph with partite sets $S$ and $T$, and let $G_{1}=G[S] \cup G[T]$ and $G_{2}$ be the graph with edges $E(B) \backslash E(G)$. Note that $e(G)=$ $e(B)+e\left(G_{1}\right)-e\left(G_{2}\right)$ and so $e\left(G_{1}\right)-e\left(G_{2}\right)=e(G)-e(B) \leqslant f(k-1, k-1)$. By Lemma 16 we have,

$$
\mathbf{x}^{T} \mathbf{x} \geqslant n\left(1-\frac{120 k^{2}}{n}\right)^{2}>n\left(1-\frac{240 k^{2}}{n}\right)=n-240 k^{2}
$$


and that $\lambda_{1}(B)=\sqrt{|S||T|}$. By Lemma 15 , also note that $e\left(G_{1}\right) \leqslant 2 k^{2}$, we obtain

$$
e(S, T)=e(G)-e\left(G_{1}\right) \geqslant \frac{n^{2}}{4}-12 k^{2}-2 k^{2}=\frac{n^{2}}{4}-14 k^{2},
$$

which implies that

$$
e\left(G_{2}\right)=e(B)-e(S, T) \leqslant|S||T|-\left(\frac{n^{2}}{4}-14 k^{2}\right) \leqslant 14 k^{2} .
$$

So, bearing in mind the inequality (1), we have

$$
\begin{aligned}
\frac{2}{n}\left\lfloor\frac{n}{2}\right\rfloor\left\lceil\frac{n}{2}\right\rceil+\frac{2 f(k-1, k-1)}{n} & \leqslant \lambda_{1}=\frac{\mathbf{x}^{T}\left(A(B)+A\left(G_{1}\right)-A\left(G_{2}\right)\right) \mathbf{x}}{\mathbf{x}^{T} \mathbf{x}} \\
& =\frac{\mathbf{x}^{T} A(B) \mathbf{x}}{\mathbf{x}^{T} \mathbf{x}}+\frac{\mathbf{x}^{T} A\left(G_{1}\right) \mathbf{x}}{\mathbf{x}^{T} \mathbf{x}}-\frac{\mathbf{x}^{T} A\left(G_{2}\right) \mathbf{x}}{\mathbf{x}^{T} \mathbf{x}} .
\end{aligned}
$$

Note that by Lemma 16,

$$
\mathbf{x}^{T} A\left(G_{2}\right) \mathbf{x}=2 \sum_{u v \in E\left(G_{2}\right)} \mathbf{x}_{u} \mathbf{x}_{v} \geqslant 2 e\left(G_{2}\right)\left(1-\frac{120 k^{2}}{n}\right)^{2} \geqslant 2 e\left(G_{2}\right)\left(1-\frac{240 k^{2}}{n}\right),
$$

therefore

$$
\begin{aligned}
& \frac{2}{n}\left|\frac{n}{2}\right|\left\lceil\frac{n}{2}\right\rceil+\frac{2 f(k-1, k-1)}{n} \\
& \leqslant \lambda_{1}(B)+\frac{2 e\left(G_{1}\right)}{\mathbf{x}^{T} \mathbf{x}}-\frac{2 e\left(G_{2}\right)\left(1-\frac{240 k^{2}}{n}\right)}{\mathbf{x}^{T} \mathbf{x}} \\
& \leqslant \lambda_{1}(B)+\frac{2\left(e\left(G_{1}\right)-e\left(G_{2}\right)\right)}{\mathbf{x}^{T} \mathbf{x}}+\frac{2 e\left(G_{2}\right) \frac{240 k^{2}}{n}}{\mathbf{x}^{T} \mathbf{x}} \\
& \leqslant \sqrt{|S||T|}+\frac{2 f(k-1, k-1)}{\mathbf{x}^{T} \mathbf{x}}+\frac{2 \cdot 14 k^{2} \frac{240 k^{2}}{n}}{\mathbf{x}^{T} \mathbf{x}} \\
& \leqslant \sqrt{|S||T|}+\frac{2 f(k-1, k-1)}{n}+2 f(k-1, k-1)\left(\frac{1}{\mathbf{x}^{T} \mathbf{x}}-\frac{1}{n}\right)+\frac{2 \cdot 14 k^{2} \frac{240 k^{2}}{n}}{n\left(1-\frac{240 k^{2}}{n}\right)} \\
& \leqslant \sqrt{|S||T|}+\frac{2 f(k-1, k-1)}{n}+2 k^{2}\left(\frac{1}{n\left(1-\frac{240 k^{2}}{n}\right)}-\frac{1}{n}\right)+\frac{2 \cdot 14 k^{2} \frac{240 k^{2}}{n}}{n\left(1-\frac{240 k^{2}}{n}\right)} \\
& =\sqrt{|S||T|}+\frac{2 f(k-1, k-1)}{n}+\frac{480 k^{4}}{n\left(n-240 k^{2}\right)}+\frac{6720 k^{4}}{n\left(n-240 k^{2}\right)} \\
& =\sqrt{|S||T|}+\frac{2 f(k-1, k-1)}{n}+\frac{7200 k^{4}}{n\left(n-240 k^{2}\right)} .
\end{aligned}
$$

Then

$$
\frac{2}{n}\left\lfloor\frac{n}{2}\right\rfloor\left\lceil\frac{n}{2}\right\rceil-\sqrt{|S||T|} \leqslant \frac{7200 k^{4}}{n\left(n-240 k^{2}\right)} .
$$


Assume to the contrary, so $|T| \geqslant|S|+2$. We consider two cases.

Case 1: $n$ is even. Since $|S|+|T|=n$, we have

$$
\begin{aligned}
\frac{2}{n}\left\lfloor\frac{n}{2}\right\rfloor\left\lceil\frac{n}{2}\right\rceil-\sqrt{|S||T|} & \geqslant \frac{n}{2}-\sqrt{\left(\frac{n}{2}-1\right)\left(\frac{n}{2}+1\right)} \\
& =\frac{n}{2}-\sqrt{\frac{n^{2}}{4}-1}=\frac{1}{\frac{n}{2}+\sqrt{\frac{n^{2}}{4}-1}}>\frac{1}{n} .
\end{aligned}
$$

So by (13), we have

$$
\frac{1}{n}<\frac{2}{n}\left\lfloor\frac{n}{2}\right\rfloor\left\lceil\frac{n}{2}\right\rceil-\sqrt{|S||T|} \leqslant \frac{7200 k^{4}}{n\left(n-240 k^{2}\right)} \leqslant \frac{8000 k^{4}}{n^{2}} .
$$

This is a contradiction for sufficiently large $n$.

Case 2: $n$ is odd. Since $|S|+|T|=n$, we have

$$
\begin{aligned}
\frac{2}{n}\left\lfloor\frac{n}{2}\right\rfloor\left\lceil\frac{n}{2}\right\rceil-\sqrt{|S||T|} & \geqslant \frac{n^{2}-1}{2 n}-\sqrt{\left(\frac{n-3}{2}\right)\left(\frac{n+3}{2}\right)} \\
& =\frac{n-\frac{1}{n}}{2}-\frac{\sqrt{n^{2}-9}}{2}=\frac{\left(n-\frac{1}{n}\right)^{2}-\left(n^{2}-9\right)}{2\left(n-\frac{1}{n}+\sqrt{n^{2}-9}\right)} \\
& =\frac{7+\frac{1}{n^{2}}}{2\left(n-\frac{1}{n}+\sqrt{n^{2}-9}\right)} \geqslant \frac{1}{n} .
\end{aligned}
$$

So by (13), we have

$$
\frac{1}{n}<\frac{2}{n}\left\lfloor\frac{n}{2}\right\rfloor\left\lceil\frac{n}{2}\right\rceil-\sqrt{|S||T|} \leqslant \frac{7200 k^{4}}{n\left(n-240 k^{2}\right)} \leqslant \frac{8000 k^{4}}{n^{2}} .
$$

This is a contradiction for sufficiently large $n$. Therefore for $n$ large enough we must have that ||$S|-| T|| \leqslant 1$.

Finally, we show that $e(G)=\operatorname{ex}\left(n, F_{k}\right)$.

Proof of Theorem 2. By way of contradiction, we assume that $e(G) \leqslant \operatorname{ex}\left(n, F_{k}\right)-1$. Let $H$ be an $F_{k}$-free graph with $\operatorname{ex}\left(n, F_{k}\right)$ edges on the same vertex set as $G$, where $S$ and $T$ induce a complete bipartite graph in $H$ (this is possible because every graph in $\operatorname{Ex}\left(n, F_{k}\right)$ has a maximum cut of size $\left.\left\lfloor n^{2} / 4\right\rfloor\right)$. Let $E_{+}$and $E_{-}$be sets of edges such that $E(G) \cup E_{+} \backslash E_{-}=E(H)$, and choose $E_{+}$and $E_{-}$to be as small as possible, i.e., $E_{+}=E(H) \backslash E(G)$ and $E_{-}=E(G) \backslash E(H)$. Since $|E(G) \cap E(H)|+\left|E_{-}\right|=e(G)<$ $e(H)=|E(G) \cap E(H)|+\left|E_{+}\right|$which implies that $\left|E_{+}\right| \geqslant\left|E_{-}\right|+1$. Furthermore, we have that $\left|E_{-}\right| \leqslant e(G[S])+e(G[T])<2 k^{2}$. Finally, by (10) we have that $\left|E_{+}\right|<15 k^{2}$. Now, by the Rayleigh quotient [19] and Lemma 16, we have that

$$
\lambda_{1}(H) \geqslant \frac{\mathbf{x}^{T} A(H) \mathbf{x}}{\mathbf{x}^{T} \mathbf{x}}=\lambda_{1}(G)+\frac{2}{\mathbf{x}^{T} \mathbf{x}} \sum_{i j \in E_{+}} \mathbf{x}_{i} \mathbf{x}_{j}-\frac{2}{\mathbf{x}^{T} \mathbf{x}} \sum_{i j \in E_{-}} \mathbf{x}_{i} \mathbf{x}_{j}
$$




$$
\begin{aligned}
& \geqslant \lambda_{1}(G)+\frac{2}{\mathbf{x}^{T} \mathbf{x}}\left(\left|E_{+}\right|\left(1-\frac{120 k^{2}}{n}\right)^{2}-\left|E_{-}\right|\right) \\
& \geqslant \lambda_{1}(G)+\frac{2}{\mathbf{x}^{T} \mathbf{x}}\left(\left|E_{+}\right|-\left|E_{-}\right|-\frac{240 k^{2}}{n}\left|E_{+}\right|+\frac{\left(120 k^{2}\right)^{2}}{n^{2}}\left|E_{+}\right|\right) \\
& \geqslant \lambda_{1}(G)+\frac{2}{\mathbf{x}^{T} \mathbf{x}}\left(1-\frac{240 k^{2}}{n}\left|E_{+}\right|+\frac{\left(120 k^{2}\right)^{2}}{n^{2}}\left|E_{+}\right|\right) \\
& >\lambda_{1}(G),
\end{aligned}
$$

for sufficiently large $n$, where the last second inequality by $\left|E_{-}\right|<2 k^{2}$ and $\left|E_{+}\right| \geqslant\left|E_{-}\right|+1$ and the last inequality by $\left|E_{+}\right|<15 k^{2}$. Therefore we have that for $n$ large enough, $\lambda_{1}(H)>\lambda_{1}(G)$, a contradiction. Hence $e(G)=e(H)$.

From the above discussion, we complete the proof of Theorem 2.

\section{Acknowledgements}

The authors would like to express their sincere thanks to the two referees for their helpful comments and suggestions of the manuscript.

\section{References}

[1] H. L. Abbott, D. Hanson, and N. Sauer. Intersection theorems for systems of sets. J. Combin. Theory Ser. A, 12:381-389, 1972.

[2] N. Alon, R. A. Duke, H. Lefmann, V. Rödl and R. Yuster. The algorithmic aspects of the regularity lemma. J. Algorithms, 16(1):80-109, 1994.

[3] A. Berman and X.-D. Zhang. On the spectral radius of graphs with cut vertices. $J$. Combin. Theory Ser. B, 83(2):233-240, 2001.

[4] B. Bollobás, J. Lee and S. Letzter. Eigenvalues of subgraphs of the cube. European J. Combin., 70: 125-148, 2018.

[5] R. A. Brualdi and E. S. Solheid. On the spectral radius of complementary acyclic matrices of zeros and ones. SIAM J. Algebraic Discrete Methods, 7(2):265-272, 1986.

[6] V. Chvátal and D. Hanson. Degrees and matchings. J. Combin. Theory Ser. B, 20(2):128-138, 1976.

[7] D. Conlon and J. Fox. Graph removal lemmas. In Surveys in combinatorics 2013, volume 409 of London Math. Soc. Lecture Note Ser., pages 1-49. Cambridge Univ. Press, Cambridge, 2013.

[8] E. R. van Dam. Graphs with given diameter maximizing the spectral radius. Linear Algebra Appl., 426(2-3): 454-457, 2007.

[9] M. N. Ellingham and X.-Y. Zha. The spectral radius of graphs on surfaces. $J$. Combin. Theory Ser. B, 78(1): 45-56, 2000. 
[10] P. Erdős, Z. Füredi, R. J. Gould, and D. S. Gunderson. Extremal graphs for intersecting triangles. J. Combin. Theory. Ser. B, 64(1):89-100, 1995.

[11] P. Erdős, P. Frankl, and V. Rödl. The asymptotic number of graphs not containing a fixed subgraph and a problem for hypergraphs having no exponent. Graphs Combin., 2(2):113-121, 1986.

[12] L.-H. Feng, P.-L. Zhang, W.-J. Liu. Spectral radius and $k$-connectedness of graphs. Monatsh. Math., 185(4):651-661, 2018.

[13] M. Fiedler and V. Nikiforov. Spectral radius and Hamiltonicity of graphs. Linear Algebra Appl., 432(9):2170-2173, 2010.

[14] J. Fox. A new proof of the graph removal lemma. Ann. of Math. (2), 174(1):561-579, 2011.

[15] Z. Füredi. Extremal hypergraphs and combinatorial geometry. In Proceedings of the International Congress of Mathematicians, Zürich, 1994, volume. 2, pages 1343-1352. Birkhäuser, Basel, 1995.

[16] Z. Füredi. A proof of the stability of extremal graphs, Simonovits' stability from Szemerédi's regularity. J. Combin. Theory Ser. B, 115:66-71, 2015.

[17] Z. Füredi and M. Simonovits. The history of degenerate (bipartite) extremal graph problems. In Erdős centennial, volume 25 of Bolyai Soc. Math. Stud., pages 169-264. János Bolyai Math. Soc., Budapest, 2013.

[18] P. Hansen and D. Stevanović. On bags and bugs. Discrete Appl. Math., 156(7):986997, 2008.

[19] R. A. Horn and C. R. Johnson. Matrix Analysis. Cambridge University Press, New York, 1985.

[20] W.-J. Liu, M. Liu, and L.-H. Feng. Spectral conditions for graphs to be $\beta$-deficient involving minimum degree. Linear Multilinear Algebra, 66(4): 792-802, 2018.

[21] V. Nikiforov. Bounds on graph eigenvalues II. Linear Algebra Appl., 427(2-3):183$189,2007$.

[22] V. Nikiforov. A contribution to the Zarankiewicz problem. Linear Algebra Appl., 432(6): 1405-1411, 2010.

[23] V. Nikiforov. The spectral radius of graphs without paths and cycles of specified length. Linear Algebra Appl., 432(9):2243-2256, 2010.

[24] V. Nikiforov. Some inequalities for the largest eigenvalue of a graph. Combin. Probab. Comput., 11(2):179-189, 2002.

[25] V. Nikiforov. Some new results in extremal graph theory. In Surveys in Combinatorics 2011, volume 392 of London Math. Soc. Lecture Note Ser., pages, 141-181. Cambridge Univ. Press, Cambridge, 2011.

[26] I. Z. Ruzsa and E. Szemerédi. Triple systems with no six points carrying three triangles. In Combinatorics (Proc. Fifth Hungarian Colloq., Keszthely, 1976), Coll. Math. Soc. J. Bolyai 18, Volume II, pages 939-945. Keszthely, 1978. 
[27] A. Sidorenko. What we know and what we do not know about Turán numbers. Graphs Combin., 11(2):179-199, 1995.

[28] R. P. Stanley. A bound on the spectral radius of graphs with e edges. Linear Algebra Appl., 87: 267-269, 1987.

[29] D. Stevanović, M. Aouchiche, and P. Hansen. On the spectral radius of graphs with a given domination number. Linear Algebra Appl., 428(8-9): 1854-1864, 2008.

[30] H. S. Wilf. Spectral bounds for the clique and independence numbers of graphs. J. Combin. Theory Ser. B, 40(1):113-117, 1986. 